\title{
Percepción que poseen estudiantes inmigrantes sobre su inserción escolar en colegios del sector Tierras Blancas de la comuna de Coquimbo
}

\section{Perception of immigrant students on their incorporation in the schools of tierras Blancas neighborhood in Coquimbo region}

\author{
Álvaro Araya Ahumada ${ }^{1}$ \\ Emma Araya Miranda ${ }^{2}$ \\ Aracely Cartes Chaparro ${ }^{3}$ \\ Bastián Morales Moyano ${ }^{4}$ \\ Constanza Oróstigue Castillo ${ }^{5}$
}

\begin{abstract}
Resumen
La presente investigación busca dar cuenta de la percepción que poseen estudiantes inmigrantes sobre su inserción escolar en colegios del sector de Tierras Blancas de la comuna de Coquimbo. En este marco, a partir de la caracterización de su proceso de inserción en la realidad educativa local y atendiendo a su opinión respecto de la realidad escolar en la que se encuentran insertos, el estudio ahonda en la percepción que entregan los entrevistados sobre su formación escolar y en las problemáticas que se les presentan en el contexto educativo. Entre los principales hallazgos encontrados se puede establecer que los estudiantes inmigrantes entrevistados poseen una valoración positiva de la institución educativa a la que pertenecen, pues destacan la exigencia, el sistema evaluativo y el buen trato docente, definiendo en términos amplios que el sistema educativo chileno representa tranquilidad y orden, en comparación a las escuelas de sus países de origen.
\end{abstract}

Palabras clave: Educación, Migración, Interculturalidad, Inclusión.

1 Profesor de Estado en Castellano y Filosofía (ULS).

Correo electrónico: a.arayahumada@gmail.com ORCID: https://orcid.org/0000-0002-4282-1064

2 Profesora de Estado en Castellano y Filosofía (ULS). Estudiante de Magíster en Estudios de Género e Intervención Psicosocial (UCEN).

Correo electrónico: arayamiranda1995@gmail.com ORCID: https://orcid.org/0000-0002-0873-5517

3 Profesora de Estado en Castellano y Filosofía (ULS).

Correo electrónico: aracely.cartes@gmail.com ORCID: https://orcid.org/0000-0002-6398-6001

4 Profesor de Estado en Castellano y Filosofía (ULS).

Correo electrónico: bpmorales64@gmail.com ORCID: https://orcid.org/0000-0002-5328-9384

5 Profesora de Estado en Castellano y Filosofía (ULS).

Correo electrónico: corostiguec@gmail.com ORCID: https://orcid.org/0000-0002-5128-5451 


\begin{abstract}
This research seeks to account for the perception that immigrant students have on their incorporation in the schools of Tierras Blancas neighborhood in Coquimbo region. In this framework, based on the characterization of their insertion process in the local educational reality, and considering their opinion regarding the school reality in which they are inserted, the study delves into the perception given by the interviewees about their school education and training, as well as the problems that arise in their educational context. Among the main findings found, it can be established that the immigrant students interviewed have a positive assessment of the educational institution to which they belong, since they highlight the demand, the evaluation system and the good teaching treatment, defining in broad terms that the Chilean educational system represents tranquility and order, compared to the schools in their countries of origin.
\end{abstract}

Keywords: Education, Migration, Interculturality, Inclusion.

\title{
Introducción
}

En la actualidad, pareciera muy difícil referirse a alguna temática social sin considerar la preeminencia de la globalización. Comprendido como un proceso de carácter complejo compuesto de múltiples interrelaciones, dependencias e interdependencias entre aparatos geográficos, políticos, económicos y culturales (Giddens en Fernández y Ruiz, 2013), dicho concepto conlleva un proceso de expansión y profundización de las relaciones sociales y de las instituciones dentro del espacio y el tiempo, de manera que las primeras resultan influidas no solo por las acciones y/o decisiones que los diferentes grupos sociales realizan, sino por hechos y acontecimientos que suceden a escala mundial. Resulta innegable, entonces, la influencia que posee este proceso en la sociedad, cuyas mayores implicancias se desarrollan en un plano económico, pues conllevan flujos comerciales de capital, información y conocimiento y que, en distintos niveles, terminan abarcando a la mayoría de los países, donde un gran porcentaje de ellos cumple un rol pasivo y vulnerable frente a los cambios producidos por estas políticas, directamente emanadas de organismos internacionales (García y Pulgar, 2010), generando la emergencia de problemáticas sociales y económicas.

La globalización desencadena notorios costos a nivel mundial al concentrarse el poder económico en algunas naciones. Así, en cuanto al ámbito laboral existe una concentración patente en aquellos países que 
poseen poder económico, produciendo entonces desempleo en las naciones restantes (Barattini, 2009). En el mismo sentido, puntualizado en el caso de América Latina, la crisis laboral ha generado precariedad, desempleo, bajas de salario, etc., potenciando el crecimiento de una economía no estructurada, dando origen a la denominada "informalización del trabajo" (Meller, 2001), donde los contratos se caracterizan por ser informales, provocando que los trabajadores se muevan geográfica y laboralmente en búsqueda de oportunidades de trabajo. Del mismo modo, se producen movimientos poblacionales de gran magnitud, como inmigraciones vinculadas a guerras, epidemias, hambre, entre otros (Mesino, 2009). Este fenómeno migratorio es entendido como la génesis de una globalización cultural, pues la globalización capitalista traslada el modo de consumo a naciones menos desarrolladas, que se ven obligadas a adquirir patrones culturales foráneos (García y Pulgar, 2010).

Desde el punto de vista de las migraciones, la globalización es compleja, pues genera impactos culturales, educacionales y políticos en las naciones, produciendo interacciones de diversa índole que incurren en la coexistencia de diferentes culturas; pese a ello, en aquellas naciones que reciben inmigrantes, la cultura nativa sigue predominando con una tendencia hacia la homogeneización de las culturas (Mesino, 2009). No obstante, la inclusión implica reconocer la existencia de características propias que persisten ante la asimilación, resistiéndose a la homogeneización y subsunción de la diversidad de culturas (Arpini, 2007). El reconocimiento de un universo multicultural implicaría la asimilación de las diferencias para generar oportunidades de diálogo, ligadas al fomento de la preservación del actuar cultural humano expresado día a día, lo que trae consigo la interrelación de una diversidad de personas dentro del contexto homogeneizador provocado por la globalización (Picotti, 2007).

La diversidad cultural, dentro del ámbito educativo, no ha sido abordada de manera consistente a partir de las políticas públicas, pues se sitúan desde una visión lejana a la interculturalidad, potenciando la homogeneización cultural (Mondaca, Rivera y Gajardo en Mondaca y Gajardo, 2015). Es así como la cultura inmigrante es encubierta por la nativa dominante, invisibilizando sus aportes y prescindiendo del diálogo entre ellas. Por esto, se requiere la contextualización del fenómeno migratorio en el ámbito educativo, en términos de atender programas que protejan los derechos del niño inmigrante, resguardando su inserción educacional, el acceso a servicios 
locales e integración cultural (Joiko y Vásquez, 2016), puesto que los estudiantes inmigrantes deben enfrentar otros obstáculos en comparación a los estudiantes nativos. En esta línea, el fenómeno migratorio implicaría una interrelación social con personas poseedoras de una cultura diferente, mediante el diálogo e interacciones que se producen dentro de la institución educativa (Díaz-Aguado en Hombrados-Mendieta y Castro-Travé, 2013).

En el plano nacional, la temática de la migración no es ajena. Es más, en el último tiempo la llegada de inmigrantes ha ascendido exponencialmente en comparación a diez años atrás, información que se ratifica a través de las cifras que entrega la Organización Internacional del Trabajo (2017), las cuales expresan que la población inmigrante creció 4,9\% por año, entre el 2010 y el 2015. Es en este último año donde el Departamento de Extranjería y Migración del Ministerio del Interior y Seguridad Pública asegura que el asentamiento de inmigrantes en Chile creció un 123\%. Estos valores han generado debate acerca de la inclusión que llevan a cabo los chilenos respecto de la población inmigrante, pues si se piensa que la inclusión consiste en percibir al otro entendiendo sus diferencias (Benavides y Galaz, 2013), se comprende que tal concepto se relaciona con el tipo de experiencia que Chile ofrece a estos nuevos grupos, en cuanto a calidad de oportunidades y condiciones sociales que promuevan su permanencia en el país (Jorge et al., 2012).

Si se consideran los lugares donde reside la población inmigrante en Chile, se puede establecer que, más que incluir a este grupo de personas en la ciudadanía chilena, lo que ocurre actualmente es una "nueva expresión de vulnerabilidad urbana" (Margarit y Bijit, 2014, p. 20) pues los inmigrantes tienden a ubicarse en determinadas ciudades del país y, dentro de ellas, suelen concentrarse en los sectores periféricos o marginales. Tal concentración genera un desequilibrio en la distribución de la población migrante, situación que queda en evidencia en el hecho de que actualmente existan en el país "escuelas públicas que por sus condiciones geográficas y culturales presentan porcentajes de inmigrantes superiores al 30, 40 o incluso 50 por ciento de la matrícula" (Jiménez et al. 2017, p. 106). Ello se constituye en una manifestación de la segregación residencial socioeconómica que reproduce y perpetúa la desigualdad social (Rodríguez y Arriagada, 2004) y que a la vez fragmenta y polariza a la ciudad (Margarit y Bijit, 2014).

En términos de política migratoria, el Estado chileno posee tendencias hacia concepciones "asimilacionistas" que se reducen a la capacidad 
para adecuarse del migrante, puesto que "solo [se busca] su adaptación a la sociedad nacional, sin respetar las identidades culturales originarias" (Alarcón, 2010, p. 128). En la historia chilena es posible reconocer oleadas de migrantes que han ingresado al país en distintas ocasiones, siendo una de las más reconocidas por el colectivo social la ocurrida en la década de los 90, cuando Chile volvía a la democracia. En aquellos años, un gran número de ciudadanos peruanos llegó a residir al país, formando parte de la realidad chilena, razón por la cual el Estado promovió leyes para controlar la entrada y salida de inmigrantes en el territorio nacional, buscando dar soluciones a algunas de las problemáticas que enfrentaban en Chile, principalmente respecto del acceso a servicios básicos e inclusión social, debido a que los ciudadanos peruanos consideraban la cultura chilena como ajena a su identidad, lo que provocó una posición pasiva en cuanto a su participación en la sociedad chilena (Alarcón, 2010).

Actualmente, Chile experimenta un nuevo proceso inmigratorio configurado por el alto número de personas haitianas que ha llegado al país. De acuerdo a las cifras proporcionadas por el Director General de Asuntos Consulares y de Inmigración, Carlos Appelgren, ante la Comisión de Relaciones Exteriores de la Cámara de Diputados (julio de 2017), fueron 44.289 los ciudadanos haitianos que ingresaron a la nación, superando así la cifra de 43.898 haitianos que se registró el año anterior (González, 2017). La llegada masiva de inmigrantes provoca la conformación de representaciones en torno a su figura, comúnmente asimilada como "el otro", motivada por las ideas que se generan en torno a ellos y que reflejan lo que la sociedad es, conformando en muchas ocasiones una visión negativa del inmigrante (Bravo, 2012). Tales imaginarios sociales se reproducen en ámbitos como la comunidad educativa, donde es el mismo alumnado quien reitera estos prejuicios y/o estereotipos, los cuales pueden volverse permanentes y sistemáticos en el tiempo (Alarcón, 2010). En consecuencia, se aparta y reduce a estas personas a su condición de inmigrantes (Pavez, 2012), pues su realidad se afronta de manera convencional, colectiva (Alarcón, 2010). 


\section{Sobre la migración}

La inmigración resulta una materia pertinente de estudio, pues es un fenómeno social que genera un impacto de carácter "complejo" a nivel mundial, derivado de las múltiples variables que influyen en el hecho de que un individuo se traslade de un país a otro (De Lucas en Acuña, 2007). La inmigración es un proceso interdisciplinario porque su estudio abarca distintas áreas sociales, siendo posible abordarlo desde la sociología, la antropología, la economía, la psicología, como también en términos culturales, sociopolíticos y educativos (Mora, 2013). El fenómeno inmigratorio conlleva una relación de interculturalidad, pues como resultado del cruce de fronteras que experimentan los migrantes se produce la coexistencia de diversas culturas en un mismo espacio social (Aguilar y Buraschi, 2012). Dicha coexistencia genera distintos desafíos a las naciones que enfrentan un fenómeno inmigratorio, pues deben dar respuesta a las nuevas exigencias económicas, socio-políticas y educativas (Pratt en Acuña, 2007) que se producen en búsqueda de la inclusión de los ciudadanos inmigrantes a la sociedad que los recibe.

Según Margarit y Bijit (2014), la inmigración se define como la base de las modificaciones culturales, en términos que, esta última, conlleva cambios culturales significativos que afectan a la sociedad a la que el inmigrante arriba, lo que ocasiona la creación de nuevas concepciones y visiones de mundo por parte de la ciudadanía nativa generando, en consecuencia, un impacto en la calidad de vida de quienes conforman la sociedad. Para Malmusi y Lopez del Vallado (2007), la inmigración es la llegada de una o más personas a un país, para radicarse en él. Siguiendo la línea de los autores, el concepto es definido como un fenómeno sociodemográfico de una alta complejidad, ya que consta de un carácter cuantitativo y uno cualitativo que tienen que ver con las características de una determinada población, las cuales a su vez estarían influenciadas por diversos factores, tales como el grado de alfabetización, la educación, la ocupación formal e informal y el nivel socioeconómico. Los autores plantean que la imagen social de un inmigrante lo define como una persona diferente, en cuanto a rasgos físicos, y que además se enfrenta problemas de pobreza y marginalidad. De acuerdo a la percepción de Penchaszadeh (2015), los extranjeros a diferencia de los inmigrantes se definen por su vinculación social hacia lo pasajero, pues se asocian a la movilidad, siendo meros espectadores, de modo que poseen un rol pasivo. Por el contrario, los inmigrantes se definen 
socialmente a través de una concepción errónea, debido a que se alejan de su realidad social en búsqueda de una aspiración mayor; no obstante, poseen un rol activo al visibilizar las debilidades del funcionamiento social, puesto que se ven obligados a abandonar sus casas, dejando atrás sus países y a buscar trabajos siendo mano de obra barata.

En términos de la importancia de las realidades migratorias, la evidencia internacional señala la relevancia de evaluar el comportamiento de un país desde la mirada de un nuevo ciudadano. A partir de la interculturalidad se lleva a cabo entonces una interrelación entre los diversos grupos que componen la sociedad, lo que en la escuela resulta en una experiencia cultural; sin embargo, para que esto se lleve a cabo es necesario conocer los alcances, a veces complejos, de tal interacción. De acuerdo a ello, el discurso y la percepción de los estudiantes inmigrantes que se integran al sistema escolar de un país devela la consecución de una educación intercultural, en el entendido que no se pueden desconocer los diversos efectos sociales que genera el fenómeno de la migración (Acuña, 2007). Así, la importancia de investigar los procesos migratorios permite visibilizar problemáticas referentes a la inclusión y distribución residencial de este nuevo grupo de personas, puesto que este fenómeno conlleva causas y consecuencias que involucran tanto a la sociedad de la cual se emigra como de aquella a la que se llega, emergiendo nuevas concepciones sobre la ciudadanía, la familia y la comunidad a la que pertenecen (Cano y Soffia, 2009).

La problematización que motiva la pregunta de investigación se vincula con la ascendencia exponencial de individuos que han decidido migrar a Chile, acto que representa un fenómeno de carácter contingente para el país, y más aún para la Región de Coquimbo. La carencia de investigaciones que abarquen las opiniones de inmigrantes respecto de su llegada y experiencias vividas en la región como, de igual manera, el desconocimiento de las percepciones que los estudiantes inmigrantes poseen sobre su inserción al sistema escolar chileno, hace pertinente que se investigue sobre este tema, pues su estudio implicaría reconocer la vinculación de la experiencia educativa de los estudiantes inmigrantes con su nuevo entorno, sobre todo si se considera que es en la escuela donde el individuo se ve en la necesidad de interactuar en el contexto de una realidad que le resulta ajena, dado que este es un espacio donde niños y jóvenes migrantes deben compartir diariamente con los individuos y con la cultura que los recibe (Hein, 2012). 
La apertura a espacios propicios para el diálogo intercultural también es una de las prioridades que motiva esta investigación; sobre ello señala Fornet-Betancourt (2004) que el concepto de interculturalidad puede ser comprendido como una disposición al diálogo abierto entre culturas distintas, donde los individuos adquieren una postura mediante la cual, en su condición de ser humano, se habitúan a la convivencia con el "otro", siendo esta relación el producto de la interacción con otras culturas, gracias a la actitud que adopta el ser humano abierto a la pluralidad. En este sentido, para Salas (2006) la apertura de una cultura a otra resulta una predisposición de la relación entre individuos que remite a modos de contacto que abordan la dimensión del "inter" como posibilidad de diálogo "entre" culturas, lo que implica abordar la experiencia humana como el producto de la diversidad de interpretaciones de mundo en el cual cada persona se ve inserta. Según Arpini (2007), la interculturalidad es el reconocimiento de los sujetos pertenecientes a diferentes culturas afrontando el desafío de integrar y aceptar sus visiones de mundo y percepciones de la realidad, lo que implicaría entender la socialización de los individuos como un diálogo entre culturas asociada a la constitución de identidades propias.

\section{Sobre la realidad escolar local}

Esta investigación es importante, pues hará visible el fenómeno inmigratorio que ocurre en Tierras Blancas, sector que se ubica en los límites periféricos de la comuna de Coquimbo y que ha sido considerado un lugar de pobreza y marginalidad social desde su origen debido a la lejanía geográfica que presenta en relación a los centros urbanos de Coquimbo y La Serena, como también por la forma en que se empezó a poblar en la década del 60, cuando un grupo de personas que se dedicaban principalmente a trabajos agrícolas o mineros decidió ocupar los terrenos del sector en búsqueda de una solución habitacional. Con el tiempo, Tierras Blancas ha logrado concentrar un alto número de residentes producto de la amplia construcción de viviendas sociales en el lugar, como también por las oportunidades laborales que ofrece el barrio industrial ubicado en sus cercanías, hechos que en la actualidad han motivado que un gran número de familias de origen latinoamericano hayan llegado a vivir a este territorio. En consecuencia, la realización de una investigación local que busque conocer la opinión de los estudiantes inmigrantes sobre su proceso de escolarización en la zona 
de Tierras Blancas no solo se hace necesaria, sino también factible debido a su masiva presencia en el sector y a la posibilidad de acceder a las instituciones educativas existentes en él.

En esta línea investigativa, es necesario comprender la Educación Municipal en palabras de Paredes y Pinto (2009), quienes establecen que, en Chile, existen dos tipos de escuelas subvencionadas: las escuelas municipales y las particulares. Las primeras dependen de la administración consistorial, mientras que las segundas son dirigidas por privados. No obstante, ambas obtienen una subvención por alumno y por su respectiva asistencia, pues de este modo se incentiva a la escuela a obtener la atracción y retención de sus estudiantes.

Para los autores Castillo et al. (2011), la educación municipal es la encargada tanto de la formación ciudadana como de la formación académica del alumnado, sin considerar para ello el estrato social del cual proviene, teniendo en cuenta la diversidad de estudiantes que asisten a escuelas municipales. Por su parte, Paredes y Pinto (2009) entienden la educación municipal como aquella que actúa acorde a los objetivos educativos que plantea cada municipio, de este modo se articula en el marco de una labor que promueve el bienestar nacional al estar asociada a la visión educativa que posee el Ministerio de turno. Por su parte, Erazo et al. (2012) entienden el concepto de educación pública como la relación de asociación entre la institución educativa con la municipalidad como herramienta para el logro de una educación sistemática. En este sentido, la noción de educación pública, desde su vínculo con las políticas de diversos niveles administrativos que actúan a nivel municipal, potencia el quehacer educativo dentro del actuar pedagógico institucional, en conformidad a las políticas públicas vigentes.

Por otra parte, se ha hecho énfasis en el papel que juega la pobreza al abordar la interrelación migración-educación. A este respecto, Romero (2002) establece que la pobreza es una situación multidimensional que se refiere a la carencia de las necesidades básicas por parte de los sujetos, noción que se define dependiendo del contexto geográfico en el cual el sujeto se sitúa; en este sentido, se constituye como una realidad que varía de acuerdo al tiempo y lugar en que se formule. Por su parte, León (2011) señala que el concepto de pobreza - desde una noción ética- es aquel en que el individuo carece tanto de recursos económicos como de respeto propio, lo que intensifica la falta de "derechos de bienestar", de tal manera que el sujeto que se sitúe en una situación vulnerable tiene dificultades para incorporarse a la 
sociedad en la que se encuentra. Bazán et al. (2011) refieren que la pobreza es la limitación económica o insuficiencia para obtener un bienestar de vida, provocando que los sujetos considerados pobres alcancen un porcentaje de mortalidad que va en aumento debido a problemas de salud generados, por ejemplo, gracias a la ausencia de nutrición. Según las autoras, la carencia económica obstaculiza el desarrollo del individuo en el contexto social, académico y laboral.

El desarrollo de esta investigación permitirá conocer el proceso de adaptación de estos estudiantes inmigrantes a la realidad escolar local, considerando su contexto sociocultural y la percepción que poseen respecto de su inclusión social en el sector de Tierras Blancas. De igual manera, buscará exponer las opiniones que tienen los estudiantes inmigrantes respecto de las diferencias de la educación que se desarrolla en sus países de origen y las exigencias que les presenta la educación chilena en cuanto a temáticas diversas como el uso del lenguaje, la comprensión del currículo nacional, la relación con sus profesores, la interacción con sus pares, las normas de conducta, la participación en la celebración de fiestas y feriados nacionales, entre otras. En esta línea, la realización de la investigación, contribuirá a comprender la influencia que ejerce la escuela en el proceso de adaptación de un estudiante inmigrante, identificando las principales problemáticas a las que se enfrentan cuando se incorporan a la educación chilena y cómo conciben su propia identidad en el contexto escolar en el sector de Tierras Blancas de la comuna de Coquimbo.

Sobre la inclusión en educación, para Blanco (2008) es la búsqueda de una mayor participación de los estudiantes dentro de la institución educativa, fomentando la aceptación de la diversidad de los alumnos desde su identidad particular. No obstante, para el autor, el concepto de inclusión en relación a educación posee un carácter polisémico con respecto al contexto geográfico, estableciendo que la carencia de inclusión provoca la segmentación tanto cultural como social. Según Infante (2010), el concepto de inclusión se define como la forma de abordar la diversidad de todos los sujetos implicados en el contexto social y educacional, respondiendo tanto a sus diferencias étnicas como de nacionalidad y género, entre otras características que escapan al proceso de enseñanza-aprendizaje. Para la autora, la práctica inclusiva en educación se entiende como el fomento de la identidad propia de los estudiantes, incorporando las diferencias y potenciando la heterogeneidad de los individuos en contextos educacionales. 
En concordancia con lo expuesto, desde la perspectiva de organismos internacionales como la UNESCO (2005) la inclusión es definida como el proceso que promueve la participación en el aprendizaje con el propósito de reducir la exclusión tanto en circunstancias culturales como socio-educativas. En este sentido, promover una visión abarcadora de los contenidos en el acto de aprendizaje se constituye como un derecho humano asociado al reconocimiento de la identidad cultural, en términos que la inclusión, asociada al ámbito educativo, es entendida como el hecho de acoger la diversidad cultural fomentando el reconocimiento de los individuos desde su identidad propia. Para los autores Jorge et al. (2012) el concepto de inclusión, tanto en el ámbito educativo como social, es la expresión de prácticas que apoyan cada manifestación de una cultura, tal como lo son sentimientos, perspectivas y subjetividades producto de la concepción de mundo propia de una comunidad. En esta línea, la inclusión es la promoción de la diversidad por parte de sociedades abiertas a la heterogeneidad social y cultural, entendiendo la inclusión educativa como la manifestación de una sociedad que garantiza una educación atenta a las diferencias.

\section{Metodología}

A partir de los antecedentes referidos, se plantea la siguiente pregunta de investigación: ¿Cuál es la percepción que poseen estudiantes inmigrantes sobre su inserción escolar en colegios del sector Tierras Blancas de la comuna de Coquimbo? Para efectos de responder a esta problemática se sugieren los siguientes objetivos específicos:

- Caracterizar su proceso de inserción en la realidad educativa local.

- Conocer la opinión de los inmigrantes respecto de la realidad escolar en la que se encuentran insertos.

- Conocer la percepción sobre su proceso de formación.

- Identificar las principales problemáticas presentes en el marco de su proceso de formación.

En el marco de un enfoque cualitativo (comprensivo-interpretativo), la investigación corresponde a un estudio cualitativo de tipo estructural. 
Canales (2006) plantea que el enfoque cualitativo está referido al orden social, buscando dar una visión holística, ya que el objeto de estudio se considera como una unidad total. A este respecto, el estudio en cuestión tuvo como finalidad investigar una situación particular para comprender un fenómeno determinado mediante un análisis e interpretación respecto de la forma en cómo actúan las y los sujetos investigados (Simons, 2011).

Para el caso en estudio se utilizó una muestra por saturación, compuesta por jóvenes inmigrantes estudiantes de un colegio municipal del sector de Tierras Blancas de la comuna de Coquimbo. Sus historias se sitúan en el año 2018, en el marco de una inserción -incipiente en unos casos y más avanzada en otros- en el modelo no solo escolar sino también cultural chileno. El género y la edad varían entre las y los entrevistados, correspondientes a hombres y mujeres, entre los 13 y 19 años. Dos personas dieron entrevistas individuales, dos fueron parte de una entrevista grupal y tres conformaron un grupo focal. En total fueron entrevistadas(os) siete estudiantes, cuyos países de procedencia son: Colombia, Venezuela, República Dominicana, Haití y Bolivia; cabe mencionar que en el último grupo focal también relataron estudiantes de Haití y Colombia. Asimismo, de las experiencias de cada entrevistado(a) no se excluyen migraciones inter e intracontinentales previas.

Para la recolección de los datos se utilizó una entrevista abierta, ya que esta se concibe como un espacio de comunicación en el que se desarrolla una experiencia personalizada entre dos sujetos, en este caso el entrevistador y el estudiante inmigrante, la que permite, en consecuencia, conocer desde el interior tanto la conducta colectiva como la individualidad de los sujetos estudiados (Delgado y Gutiérrez, 1999).

Finalmente, para llevar a cabo el análisis de la información se utilizó la técnica de análisis de contenido. El análisis de contenido implica organizar los datos referidos en la investigación mediante un ejercicio de codificación de la información, permitiendo de esta manera conocer las percepciones de los sujetos implicados (Mayan, 2001).

Acerca del método empleado es necesario aclarar que bajo ninguna circunstancia ha pretendido velar por una visión universalizadora de la experiencia migratoria en nuestro país, en el entendido que cada experiencia de vida es única y que, por tanto, estará sujeta tanto a la contingencia local como a proyectos y motivaciones personales. Cabe señalar que, desde sus 
inicios, el trabajo buscaba dar cuenta de una situación desde una mirada respetuosa con las personas implicadas, sobre todo tomando en consideración que las realidades presentadas resultaban, en ocasiones, ajenas a las de las y los entrevistadores. En este aspecto, la intervención fue una oportunidad para crear entornos dialógicos donde las conversaciones fueran gratas y una adaptación fiel de las perspectivas en las que coincidían procesos de emigración con los de desarrollo de la identidad personal en el contexto escolar.

\section{Resultados}

En el marco de los hallazgos del estudio, se construyeron las siguientes categorías:

- Características de los estudiantes.

- Representación de la institución escolar.

- Concepción de inclusión e integración.

- Proyecciones a corto y mediano plazo.

\section{Primera categoría: característica de los estudiantes}

Los discursos de los estudiantes inmigrantes entrevistados rescatan la experiencia del sujeto como un elemento fundamental para determinar los efectos producidos en ellos a causa de su arribo al país. Algunos entrevistados expresan que han tenido dificultades para acostumbrarse, debido a que la llegada a Chile les significó un cambio radical en sus vidas:

"Lo mío fue muy radical, muy de la noche a la mañana me vine aquí. Algún día tenía que pasar eso y bueno, lo entendí.” (FG1: 44) ${ }^{6}$ “¿Cómo fue mi experiencia?... ¡Ay! Bueno, ahí yo me sentía muy asustada, por el colegio y todo. No quería nada, no quería salir, no quería hacer amigos, no quería nada." $(\mathrm{E} 1: 14)^{7}$

“(...) Quizá cosas malas son el ambiente, al menos yo, no me adapto y no me gusta estar aquí.” (FG1: 21)

6 FG1: Focus Group $\mathrm{N}^{\circ} 1$.

7 E1: Entrevista $N^{\circ} 1$. 
A partir de algunos discursos recogidos se puede establecer que algunos de los estudiantes inmigrantes entrevistados asocian su llegada a Chile con el hecho de tener que acostumbrarse a las nuevas condiciones ambientales y climáticas propias del país y, específicamente, de la ciudad de Coquimbo, las cuales son distintas a las existentes en su país de origen:

"Bien, no sentí tanto frío (risas), ya me estoy acostumbrando un poco. Es que allá hace puro calor.” (E3:30) ${ }^{8}$

En general, los estudiantes entrevistados residen en familias numerosas, compuestas por padres, hermanos y, en ocasiones, incluso por otros familiares, como tíos y primos.

En cuanto al nivel educativo de los miembros del grupo familiar, se desprende que la mayoría de los padres o cuidadores de los entrevistados poseen estudios incompletos, o bien es un tema desconocido por ellos:

"Mi mamá creo que no terminó el estudio. Mi padrastro no sé." (E3: 25)

“(...) yo no sé nada de mi padre.” (FG1:38)

“En Colombia mi mamá llegó hasta octavo, pero aquí eso es como primero medio. Bueno mi papá hasta segundo medio. Fabián, mi padrastro con quien vivo, llegó hasta quinto año en Colombia, pero no sé muy bien qué curso es ese en Chile.” (E1: 10)

El contexto social de los estudiantes entrevistados está determinado principalmente por el entorno físico donde viven puesto que, en general, existe una cercanía entre el lugar donde residen y el colegio al que asisten. Tal cercanía, contribuye a que el comportamiento de los entrevistados se vea condicionado por el entorno del barrio y la conducta que poseen sus vecinos, ya que estos representan su principal foco de socialización fuera de la institución escolar:

"Ah... bien, muy bien. Nos llevamos muy bien, de hecho, salimos a paseos con los vecinos, con las vecinas. Y bueno... eh... todos son chilenos." (E1:16)

"Solo que algunas veces algunos son medios racistas, pero en el colegio a mí no, pero en la calle sí. Porque en el colegio cuando me 
hacen algo, ahí los profesores altiro lo retan a los niños, pero en la calle a veces me hablan un poquito mal." (E3:12)

"Bueno, en mi caso, donde yo vivo ningún vecino saluda a nadie, bueno algunos. A veces mi hermano chico juega con los vecinos, pero yo no." (FG1:52).

Respecto del proceso de enseñanza-aprendizaje, las respuestas que entregan los alumnos entrevistados evidencian percepciones diferentes sobre el proceso de enseñanza- aprendizaje que tenían en su país de origen y el que reciben en Chile, señalando que actualmente perciben la inclusión de distintas culturas en las asignaturas que cursan. Además, expresan que en Chile sus aprendizajes han aumentado, pues han podido adquirir diversos conocimientos que antes no tenían:

“(...) Los profesores y las autoridades son súper atentos, entonces tratan de que las asignaturas agreguen todas las culturas.” (E2:8)9

“(...) Acá me gusta más, porque allá, cuando estaba en República Dominicana, no sabía leer. Me costaba mucho, y como acá ya sé muchas cosas. Lo malo es que allá no sabía muchas cosas, y acá ya aprendí la mayoría de lo que no sabía. Aquí aprendí a leer, pero allá no sabía leer.” (E3: 9)

Así mismo, en cuanto al rendimiento de los estudiantes y el proceso de adaptación a un nuevo colegio, se evidencia un frecuente contraste entre el colegio actual y el de su país de origen. Sin embargo, la percepción sobre el rendimiento académico no siempre es positiva, ya que algunos estudiantes señalan presentar dificultades en diversas asignaturas y complejidad en el manejo de estas, lo que obstaculiza su rendimiento académico:

“(...) Ahora que me cambié, un poquito más mal que en el otro, pero en general bien porque sí. Además, que he llevado muy poquito tiempo en el colegio entonces todavía no sé las notas (...).” (E2:10)

“(...) Cuando no lo comprendo comienzo a llorar en la sala, porque algunas veces me da rabia que no comprendo y me molesta." (E3:72) 
En otro sentido, los entrevistados se muestran mayoritariamente indiferentes respecto a la influencia que podría generar la cultura del país sobre su proceso de enseñanza-aprendizaje e, incluso, en algunas declaraciones se establece que no hay relación entre la cultura y el aprendizaje que pueden alcanzar como estudiantes:

“(...) No, porque no sé, como que, me da lo mismo eso (...)." (E2:12)

"Para mí no, porque el aprendizaje de los niños depende de cada uno. Si ellos quieren aprender. Ellos son los que deciden si quieren aprender y poner atención o no.” (E1: 46)

\section{Segunda categoría: representación de la institución escolar}

De acuerdo a los discursos recogidos, la percepción que tienen los estudiantes entrevistados sobre la institución escolar a la que asisten actualmente en Tierras Blancas es positiva y denota una constante comparación de su actual escuela con la que asistían en su país de origen, aun cuando las preguntas no necesariamente iban dirigidas a establecer una comparación entre tales instituciones.

En términos generales, los entrevistados asocian su institución educativa con la idea de tranquilidad y buen trato que reciben de la comunidad escolar, lo que se puede evidenciar con las siguientes respuestas:

“(...) A mí me gusta porque es tranquilo, es como más tranquilo que donde yo estaba, sin bulla, ahí tranquilo, sin problema (...)." (E3.: 2)

"Es más tranquilo que el de allá (Santo Domingo), porque allá te hacen bullying y acá no (...).” (E3: 8)

“(...) Desde que yo llegué a Chile solo he estado en este colegio, lo otro sería compararlo con los de Colombia y hay mucha diferencia, porque, por ejemplo, allá los alumnos les faltan más el respeto a los profesores que acá. Eso noté cuando yo llegué, porque acá las salas eran muy silenciosas comparadas con las de Colombia, por ejemplo, en Colombia solo llegaban y se paraban, sin permiso, sin nada, empezaban a hacer desorden, es mucha la diferencia. Entonces yo me tuve que adaptar porque yo era muy desordenado". (FG1: 77) 
Cuando los estudiantes responden a interrogantes en que necesariamente deben comparar su escuela de origen con la que se encuentran actualmente, optan principalmente por evidenciar las dificultades que enfrentan en el proceso de aprendizaje debido a la diferencia de las asignaturas y los contenidos tratados en su escuela actual y su escuela de origen:

“(...) hoy día: me saqué un 1.0 en una prueba de música, y me enojé, me dan ganas de llorar, pero nadie lo sabe. Porque allá yo no hacía música, es primera vez que yo estoy haciendo música, acá, por eso me saqué un 1.0" (E3: 82).

En el mismo sentido, debido a que de forma recurrente constatan que son menores las exigencias educativas en su país de origen en comparación a la formación que reciben en Chile, se vuelve drástico el cambio, de esta manera señalan que inglés es una de las asignaturas más difíciles, debido a que pasan de tener una educación de un nivel más básico a una de carácter superior y por tanto más exigente:

"En inglés siempre doy una excusa para que me saquen los profesores. Es que es como, cuando el profesor está hablando, es como si fuera "él sabe que tú sabes también" y la cosa no es así. Cuando, por ejemplo, él te dice "diga eso" es como si fuera "tú lo sabes" como cuando él está hablando inglés los niños lo comprenden, pero yo no lo comprendo, y yo digo ¿cómo ellos lo comprenden y yo no? Ya no hago inglés, me quitaron inglés, pero hoy día voy a ver cómo me va, me quedé en reforzamiento y no me gusta (...)” (E3: 81).

"Por ejemplo, una materia, la de inglés, aquí el profesor habla perfectamente y en Bolivia no, nos daban traductor para traducir y esas cosas" (FG1: 9)

“(...) Porque a veces unas palabras no entiendo, porque todo el tiempo habla en inglés a comparación como les dije de Bolivia, el inglés no, es más el idioma que tiene Bolivia, el quechua.” (FG1: 131).

No obstante, pese a las dificultades que significa la asignatura de inglés en su proceso de formación, otros estudiantes entrevistados destacan y valoran el hecho de aprenderlo debido a su gran aplicabilidad, señalando además la importancia de aprender más lenguas, ya sea para ampliar el bagaje cultural o para satisfacer las necesidades de contingencia social. 
“(...) Yo estuve en México, en idiomas no solo tenían el inglés, sino que varios idiomas, a mí me gustaría que tuvieran otros idiomas como se hace en México (...)” (FG1: 149).

“(...) Si, bueno, me gustaría conocer, por ejemplo, el idioma de los mapuches, conocerlos (...)” (FG1: 151).

"Deberíamos aprender francés o portugués, porque ahora en este tiempo han llegado muchos inmigrantes haitianos y todo eso, entonces para ellos les cuesta mucho cuando van a conseguir empleo o cuando necesitan comprar alguna cosa solo hablan en francés o portugués" (E1: 40)

A través de las declaraciones recogidas, los entrevistados aluden a actos de violencia en el trato que los profesores protagonizan en el país del cual provienen y que -según sus percepciones- no son comunes en Chile. Esto hace que, en términos amplios, los entrevistados manifiesten una buena percepción de sus profesores, considerando el trato que reciben de ellos:

“(...) A mí me va bien, pero algunas veces si hago algo me asusto, y ahí me dicen "no te asustes que no te voy a hacer nada (...)". (E3: 57)

"Yo creo que los profesores acá son más o menos, de allá algunos maltratan a los niños y les pegan... yo me acuerdo de que un profesor me pegó y acá no pasa eso" (FG1: 106)

Al mismo tiempo, la buena percepción del ejercicio docente es fundamentada en la forma en que los profesores enseñan, puesto que los entrevistados aprecian el hecho de que sus actuales educadores atiendan a los detalles y lo importante de cada asignatura, como también que expliquen los contenidos en reiteradas ocasiones:

"Yo creo que los profesores explican bien, pero algunos como allá en mi país, los profesores si te dicen la cosa ahora como no explican dos veces, acá a veces yo voy al lado de los profesores y son como 50 veces". (FG1: 111)

"Yo creo que, sí explican muy bien los contenidos. Explican muy bien en detalles y lo bueno es que también enseñan lo más importante de cada asignatura" (FG1: 112) 
Sin embargo, una de las críticas que realizan algunos de los entrevistados a sus actuales docentes tiene relación con la rapidez con la que hablan los profesores al momento de realizar la clase, lo que dificulta la comprensión de los contenidos. Además, se destaca el hecho de que no siempre los profesores son conscientes de que hablan rápido, sino hasta que los estudiantes se lo señalan:

“(...) lo que no me gusta (de los profesores) es que hablan muy rápido $(\ldots) "(\mathrm{E} 1: 28)$.

“(...) En mi colegio, en la sala cuando el profesor está hablando, la profesora... no comprendo, y a veces ellos me dicen "¿yo hablo rápido?" y yo les digo "Sí” (...)” (E3: 70)

En cuanto a la relación de los entrevistados con sus compañeros de colegio, existe una buena percepción respecto a la forma en que los recibieron cuando se incorporaron a la escuela actual. Los discursos recogidos, rescatan principalmente la ayuda que obtuvieron de parte de sus compañeros al momento de llegar a su escuela actual:

“A mí me fue bien, pero, cuando vine el primer día, vine con mi uniforme, y después mañana llegué y los niños me hicieron una sorpresa: cuando abrí la puerta -me vine con un cuaderno nomás y un lápiz, porque mi mamá no alcanzó a comprar los materiales- y ahí cuando llegué me regalaron 20 cuadernos, me regalaron una mochila nueva, lápiz, de colores, muchas cosas, materiales de colegio, me regalaron todo. Calificación: Un 7.0.” (E3: 41)

“A mí no me pasó algo así, pero me saludaron y me comenzaron a enseñar lo que dieron del otro... lo que dieron ayer, porque yo no estaba ahí, y también me estaban diciendo los nombres de los muchachos, para conocerlos. Y también me estaban diciendo cómo son los profesores. Calificación: Buena." (E3: 42)

Sin embargo, esta percepción cambia drásticamente cuando se refieren a la relación que tienen con sus compañeros una vez que ya llevan más tiempo en la institución escolar. Evidencian cambios en el comportamiento de sus compañeros, manifestando también algunas diferencias que identifican entre los compañeros de la escuela del país de donde provienen y los que tiene en la actualidad. 
“(...) comienzan bien contigo y después te dan banda, como si fuera ya no te conocen, o que, si yo estoy hablando contigo, o por ejemplo: somos amigos tuyos hoy día, y mañana es como que te ven y no te pescan. Y no me molesta, y no digo nada porque en el colegio yo me siento sola, y los niños dicen "¿por qué te sientes sola?". Porque allá es como normal que entre los niños se habla, se juega, se juntan en todas partes, son como hermanas, pero aquí es como hoy día soy tu amigo y mañana es como ya no te conocen (...)" (E3: 52)

“(...) allá en Colombia eran más atrevidos, les gustan decir más las cosas. Son más directos que mis compañeros de acá porque acá tratan de ocultarlo o tratan de decirlo indirectamente (...)" (FG1: 96)

En cuanto a la percepción de los métodos evaluativos existentes en el sistema escolar chileno, las y los entrevistados asocian el sistema evaluativo con la rendición de pruebas de conocimientos $y$, pese a que reconocen ciertas diferencias con el sistema de su país de origen, en términos generales se muestran conformes con el hecho de tener que rendir pruebas para evaluarse y que su rendimiento se traduzca en una calificación:

"Yo opino que sí, es muy conveniente, por ejemplo, en Colombia no hay pruebas (...) a veces nos hacen hacer algún trabajo que se hace en una clase, entonces acá lo bueno es que hay pruebas y bueno, yo me había perdido cuando era una nota al libro o cuando no, en cambio acá si me entero porque hay pruebas, entonces ahí ya yo mejoro" (FG1: 159).

“(...) A mí me gusta la forma como le dan las pruebas a todos, como la dan en cada mes, como allá le daban, como allá este mes que viene, tienes que dar la pruebas, todas las pruebas como 20 pruebas, todas juntas. Un día o a veces, todos los días, pero a mí me gusta acá como es (...)" (FG1:160).

“(...) Estoy de acuerdo con las pruebas de la escuela. Porque está bien acá como la enseñan. Por eso, está bien que pongan notas. (...)" (E1: 44). 


\section{Tercera categoría: concepción de inclusión e integración}

En primera instancia, los entrevistados expresan una clara dificultad para distinguir la diferencia entre los conceptos "inclusión" e "integración", esto se evidencia por el conflicto que surge al momento de intentar definir ambos términos, siendo "integración", en consecuencia, considerada un sinónimo de "inclusión”, generando respuestas confusas y ambigüedades en los términos.

"Me suena integrar, sí, pero la otra no.” (E3:107)

"La integración es como... ¡rayos!, ¡cómo lo digo! La integración es más como involucrarse con más cosas, participar con más gente." (E1:48)

"Integración, es como hacer que algún desconocido se integre al colegio, como en nuestro caso, aquí nos integran." (FG1: 57)

"Para mí la inclusión es incluir, es como no sé, como agregar a una persona o hacerla sentir cómoda. Para mí integrar, es lo mismo que la otra, como que no sé." (E2: 14)

Por lo tanto, los discursos emanados demuestran, inicialmente, dificultad para definir integración e inclusión, para, luego, asociar la inclusión con la participación, lo que implica identificar las siguientes percepciones:

"Como intervenir, como interesar en una cosa, como participar en baile y en esas cosas." (E3: 106)

"Me siento incluida porque me gusta participar en todo: talleres, academias, en todo lo que hacemos en el liceo.” (E1:50)

Teniendo esto en consideración, se puede apreciar una valorización sobre inclusión ligada a la calidad sobre la educación, donde, acerca de las percepciones del ejercicio docente, junto al comportamiento de los compañeros de curso dentro y fuera del aula, se rescata una valoración positiva acerca de la inclusión que reciben por parte de estos, lo que provoca, de esta manera, una percepción positiva respecto a las nociones de inclusión al momento de interactuar en el contexto escolar.

"Cuando hacemos grupos me integran a ellos." (FG1: 55)

"Los profesores me brindan apoyo en todo lo que necesito (...)" (FG1: 61) 
La mayoría de los estudiantes se inclinó por esta imagen positiva acerca de la inclusión en sus respectivos establecimientos, siempre en virtud de la óptima relación con los agentes de la comunidad educativa, tales como: directivos, profesores, apoderados, alumnos, entre otros. Además, destacan el trato privilegiado y prioritario que reciben los estudiantes extranjeros, lo que se explica por el énfasis hacia el respeto, actitudes y valores de los estudiantes que establecen las instituciones educativas.

“(...) aparte de ser el mejor el colegio de excelencia académica y mejor rendimiento, los alumnos son súper respetuosos y educados y se les recalcan los valores.” (E2: 18)

"Sí, bien, es que ellos me apoyan en todo, sí, cuando no sé algo y voy a preguntar, siempre me ayudan.” (FG1:63)

“(...) siempre han tratado como con privilegio a los extranjeros porque siempre como que nos dan la prioridad y sí, súper cómoda y todo." (E2: 16)

De este modo, por el hecho de que las visiones comunes sobre inclusión sean de carácter positivo, se desprende la tesis de que los estudiantes inmigrantes reconocen una buena imagen respecto a su inclusión al sistema escolar chileno, puesto que, en la mayoría de los casos, se presentan nociones de inclusión asociadas a la buena calidad percibida sobre la educación en torno a temáticas integradoras.

"Bien, o sea es que te tratan como uno más." (FG1: 69)

"Yo creo que sí me incluyo porque, porque me siento a gusto con el sistema que tiene Chile." (FG1: 59)

Ahora bien, existe conocimiento errado acerca de los conceptos inclusión e integración. La noción que poseen los estudiantes inmigrantes se encuentra referida a la participación o hacia la idea de ser partícipes de diversas instancias al interior del colegio en que se encuentran. La gran mayoría de discursos gira en torno a la buena relación que se tiene con los pares, docentes y otros miembros de la comunidad escolar, por lo que la imagen de inclusión es catalogada como buena, excelente, o calificada con el equivalente a la máxima nota y a su correcto cumplimiento o ejecución; sin embargo, esta apreciación es referida al carácter participativo que advierten en su calidad de estudiantes y no a una acepción acertada o exacta del término, lo que se traduce, en resumidas cuentas, en una noción 
distorsionada acerca de lo que entienden por su inclusión al sistema escolar chileno.

"Nos incentiva a participar en actividades." (E3: 115)

"Pues sí, porque participa todo el colegio. O sea, cuando hay una actividad y hay que participar en algo, todos participan y en todo." $(\mathrm{E} 1: 52)$

En cuanto a la calidad percibida sobre la educación en torno a las temáticas integradoras, si bien el reconocimiento de los conceptos, debido a su propia indefinición, resultan erráticos, tanto la integración como la inclusión se ligan, a fin de cuentas, a aquellos factores que demuestran la participación y buen trato que han sentido respecto a su inserción al aula desde su llegada al país, siendo este último punto un punto de vista favorable que se repite constantemente y que permite desprender una imagen positiva, tanto de la representación del país como del sistema escolar en el que se han visto insertos.

"Yo sí, he visto muchos extranjeros salir del colegio y he visto como el colegio le ha brindado ese apoyo para que se puedan adaptar bien sin complicaciones." (FG1:72)

"Sí, porque si no fuese así yo no estuviera aquí." (FG1: 67)

\section{Cuarta categoría: proyecciones a corto y mediano plazo}

A grandes rasgos en lo que respecta a las proyecciones a corto y mediano plazo de los estudiantes inmigrantes entrevistados, se establece que la experiencia de emigrar de un país y posicionarse en Chile, accediendo así a un mejor sistema educativo, provoca en ellos la idea de que pueden acceder a un mejor nivel de vida, de modo que creen en la movilidad social, considerando a la educación como la vía para lograrlo.

La proyección de los estudiantes y la continuación de su educación a través de la enseñanza superior es un fenómeno que tiende a repetirse entre los entrevistados, donde algunos, si bien reconocen a la educación superior como forma de movilidad social, no tienen claro sus intereses. 
“(...) Para el futuro... pues yo quiero salir de la universidad. Eh quiero trabajar, pero no sé qué quiero estudiar, por mí estudiaría todo." (E1:54)

Otros, por su parte, reconocen claramente su inclinación y con decisión asumen la continuidad de estudios con carreras tales como medicina, periodismo, etc., las cuales responden a sus gustos personales, aplicándole además utilidades a su vida cotidiana. No obstante que conciben a la educación chilena como un medio para alcanzar sus objetivos, ninguno expone con claridad la idea de radicarse en Chile, y es más evidente la visualización en otro país, que tampoco se corresponde con el de origen.

"Llegar a la universidad, estudiar el japonés y en 10 años más me gustaría ir a Japón" (FG1: 195)

En fin, se observan dudas respecto a su futuro a largo plazo, puesto que no hay claridad con relación a establecerse en un país, Chile en este caso. Lo que se vuelve evidente son las proyecciones a mediano plazo, pues todos coinciden en que quieren seguir estudiando en la educación superior, como un anhelo, a pesar de las confusiones respecto a qué estudiar, casi como un deber.

\section{Conclusiones}

Sobre la percepción que tienen los estudiantes inmigrantes respecto a su proceso de formación, en términos generales es positiva por múltiples razones, entre ellas la tranquilidad que las instituciones educativas les evocan, debido a la ayuda y apoyo que se les otorga. A esto se le suma la complejidad percibida en torno al sistema educativo, lo cual les parece apropiado para su proceso de formación, pues reconocen que aprenden más debido a exigencias que consideran mayores realizando un contraste con la educación recibida en sus países de origen. De la educación recibida en Chile destacan la labor docente como muy atenta a sus necesidades educativas, con gran disponibilidad a la resolución de dudas. En lo que respecta al sistema de evaluación existe una concordancia en cuanto a la valoración positiva en relación al método empleado en la evaluación del proceso de enseñanza-aprendizaje, señalando que las pruebas significan para ellos algo más sistemático y periódico. 
Pese a ello, los estudiantes inmigrantes entrevistados también expresan problemáticas ligadas, en primera instancia, a su proceso de adaptación al momento de arribar al país, debido al cambio cultural, pues si bien hay similitudes entre las culturas, la inmigración es un cambio radical, una transformación en la rutina. Si bien, por un lado, destacan de forma positiva las exigencias educativas que les genera el sistema educativo chileno, también les significa una problemática puesto que se ven enfrentados a asignaturas que los obligan a poner un mayor esfuerzo para alcanzar buenos resultados en su proceso de enseñanza-aprendizaje. Otra problemática se percibe en los vínculos con sus compañeros chilenos, los cuales no llegan a ser profundos ni estables.

Expresan a su vez problemáticas con el lenguaje, señalando que el chileno habla demasiado rápido, con modismos, etc., lo cual dificulta su proceso de adaptación y de comprensión, influyendo considerablemente en las relaciones que forjan.

En términos generales, respecto a la calidad percibida sobre la educación en torno a las temáticas integradoras, los estudiantes presentaron dificultades iniciales para distinguir la diferencia entre inclusión e integración, para luego expresar una valoración positiva acerca de la inclusión que reciben por parte de los diferentes agentes de las instituciones educativas, principalmente producto de la asociación del término inclusión como participación. A su vez, teniendo en cuenta las diversas actividades que ofrece el sistema escolar chileno, en las que se han visto partícipes y de las que se les incentiva a formar parte, la percepción de inclusión relacionada al trato privilegiado y prioritario que reciben los estudiantes extranjeros es valorada de buena manera, lo que se evidencia en los discursos emanados que ahondan en el sentimiento de verse involucrados, de sentirse a gusto en la institución en que estudian, expresando que, básicamente, se sienten incluidos y que, al sentirse "como uno más", como miembros que son parte de la comunidad escolar, las visiones comunes de los estudiantes inmigrantes entrevistados se traducen, en resumidas cuentas, en una percepción positiva respecto a su inclusión a los distintos colegios a los que pertenecen los estudiantes inmigrantes entrevistados del sector de Tierras Blancas en la comuna de Coquimbo. 


\section{Referencias bibliográficas}

Acuña, R. (2007). Inmigración: Exigibilidad, prestación y vulneración de Derechos Humanos o fundamentales. [Memoria para optar al Grado Académico de Licenciado en Ciencias Jurídicas y Sociales, Universidad Austral de Chile, Facultad de Ciencias Jurídicas y Sociales]. http://cybertesis.uach.cl/tesis/uach/2007/fja189i/doc/fja189i.pdf

Aguilar, M. y Buraschi, D. (2012). El desafío de la convivencia intercultural. REMHU: Revista Interdisciplinar da Mobilidade Humana, 20(38), 27-43. http://www.scielo.br/pdf/remhu/v20n38/a03v20n38.pdf

Alarcón, G. E. (2010). Las políticas inmigratorias de Chile en el área educativa. El caso de los peruanos en Chile. [Tesis para optar al grado de Magíster en Estudios Internacionales, Universidad de Chile, Instituto de Estudios Internacionales]. http://repositorio.uchile.cl/bitstream/ handle/2250/110839/alarcon_g.pdf?sequence=3

Arpini, A. (2007). Diversidad y conflictividad. Contribuciones a la formulación de categorías heurísticas para la integración y el diálogo intercultural. En: Herceg, J. (comp.). Integración e Interculturalidad (pp. 13-30). Colección idea.

Barattini, M. (2009). El trabajo precario en la era de la globalización. ¿Es posible la organización? Polis, 8(24), 17-37. http://dx.doi.org/10.4067/ S0718-65682009000300002

Bazán, A., Quintero, M. y Hernández, A. (2011). Evolución del concepto de pobreza y el enfoque multidimensional para su estudio. Quivera, 13(1), 207-219. http://www.redalyc.org/articulo.oa?id=40118420013

Benavides, M. A. y Galaz, K. V.(2013). Realidad de niños yniñas inmigrantes en Chile y la Integración en la educación. [Tesis para optar al grado de Licenciado en trabajo social, Universidad Academia de Humanismo Cristiano, Escuela de Trabajo Social]. http://bibliotecadigital. academia.cl/xmlui/bitstream/handle/123456789/1882/ttraso385. pdf? sequence $=1 \&$ is Allowed $=\mathrm{y}$

Blanco, R. (2008). Marco conceptual sobre educación inclusiva. "La educación inclusiva: El camino hacia el futuro". Conferencia Internacional de educación, Cuadragésima octava reunión. UNESCO, llevado a cabo en Ginebra. http://www.ibe.unesco.org/fileadmin/user upload/Policy_Dialogue/48th_ICE/CONFINTED_48-3_Spanish.pdf 
Bravo, R. (2012). Inmigrantes en la escuela chilena: ciertas representaciones para ciertas políticas en educación. Revista latinoamericana de educación inclusiva, 6(1), 39-52. http://www.rinace.net/rlei/numeros/vol6-num1/art2.pdf

Canales, M. (2006). Metodología de la investigación social. LOM

Cano, V. y Soffia, M. (2009). Los estudios sobre migración internacional en Chile: apuntes y comentarios para una agenda de investigación actualizada. Papeles de población, 15(61), 129-167. http://www.scielo.org.mx/pdf/pp/v15n61/v15n61a7.pdf

Castillo, P., González, A. y Puga, I. (2011). Gestión y efectividad en educación: evidencias comparativas entre establecimientos municipales y particulares subvencionados. Estudios pedagógicos, 37(1). http://dx.doi.org/10.4067/S0718-07052011000100010

Delgado, J. y Gutiérrez, J. (1999). Métodos y técnicas cualitativas de investigación en ciencias sociales. Síntesis.

Departamento de Extranjería y Migración del Ministerio del Interior y Seguridad Pública. (2015). Migración en Chile 2005-2014. http:// www.extranjeria.gob.cl/media/2016/06/Anuario.pdf

Erazo, M., Villalta, M. y Morales, M. (2012). Representaciones sociales del asociativismo municipal en educación: un marco conceptual para la educación chilena. Polis, 11(31), 141-156. http://dx.doi.org/10.4067/ S0718-65682012000100008

Fernández, M. y Ruiz, J. (2013). El concepto globalización: ¿Poblamiento o erosión semántica? La semántica de la globalización en el diario La Tercera, 1999 y 2004. Atenea, (507), 133-147. http://dx.doi.org/10.4067/S0718-04622013000100009

Fornet-Betancourt, R. (2004). Crítica intercultural de la filosofía latinoamericana actual. Trotta.

García, J. y Pulgar, N. (2010). Globalización: aspectos políticos, económicos y sociales. Revista de ciencias sociales, 16(4), 709-720. http://ve.scielo. org/scielo.php?script=sci_arttext\&pid=S1315-95182010000400014

González, V. (2 de agosto de 2017). En solo siete meses la inmigración haitiana a Chile ha superado el total del año pasado. El Mercurio. https://www.elmercurio.com/blogs/2017/08/02/53068/ 
En-solo-siete-meses-la-inmigracion-haitiana-a-Chile-ha-superado-eltotal-del-ano-pasado.aspx

Hein, K. (2012). Migración y transición: hijos de inmigrantes de origen latinoamericano en su transición de la escuela al trabajo en Chile. Si Somos Americanos, 12(1), 101-126. http://dx.doi.org/10.4067/S0719-09482012000100005

Hombrados-Mendieta, I. y Castro-Travé, M. (2013). Apoyo social, clima social y percepción de conflictos en un contexto educativo intercultural. Anales de psicología, 29(1), 108-122. https://doi.org/10.6018/analesps.29.1.123311

Infante, M. (2010). Desafíos a la formación docente: Inclusión educativa. Estudios Pedagógicos, 36(1), 287-297. http://dx.doi.org/10.4067/S0718-07052010000100016

Jiménez, F., Aguilera, M., Valdés, R. y Hernández, M. (2017). Migración y escuela: Análisis documental en torno a la incorporación de inmigrantes al sistema educativo chileno. Psicoperspectivas, 16(1), 105-116. https:// doi.org/10.5027/psicoperspectivas-vol16-issue1-fulltext-940

Joiko, S. y Vásquez, A. (2016). Acceso y elección escolar de familias migrantes en Chile: "No tuve problemas porque la escuela es abierta, porque acepta muchas nacionalidades". Calidad en la educación, (45), 132-173. http://dx.doi.org/10.4067/S0718-45652016000200005

Jorge, M., Sánchez, L. y Herrera, M. (2012). Políticas de inclusión en la formación docente: Las trayectorias culturales de los alumnos en los institutos de formación. Cuadernos de la Facultad de Humanidades y Ciencias Sociales, (43), 119-139.

http://www.scielo.org.ar/pdf/cfhycs/n43/n43a08.pdf

León, F. (2011). Pobreza, vulnerabilidad y calidad de vida en América Latina. Retos para la bioética. Acta Bioethica, 17(1), 19-29. http://dx.doi.org/10.4067/S1726-569X2011000100003

Malmusi, D. y Lopez del Vallado, J. (2007). Recomendaciones para la investigación e información en salud sobre definiciones y variables para el estudio de la población inmigrante de origen extranjero. Rev. Esp. Salud Publica, 81(4), 399-409.

$\mathrm{http}: / /$ scielo.isciii.es/pdf/resp/v81n4/original5.pdf 
Margarit, D. y Bijit, K., (2014). Barrios y población inmigrante: El caso de la comuna de Santiago. Revista INVI, 29(81), 19-77. http://dx.doi. org/10.4067/S0718-83582014000200002

Mayan, M. (2001). Una introducción a los métodos cualitativos: Un módulo de entrenamiento para Estudiantes y profesionales. Qual Institute Press. https://sites.ualberta.ca/ iiqm/pdfs/introduccion.pdf

Meller, P. (2001). Beneficios y costos de la globalización: Perspectiva de un país pequeño (Chile). Serie de estudios Socio/Económicos. (9), 1-24. http://www.cieplan.org/wp-content/uploads/2019/12/serie-estsocioec-9.pdf

Mesino, L. (2009). La globalización económica y sus implicaciones socioculturales en América Latina. Revista de Ciencias Sociales, 15(1), 126-138. http://www.scielo.org.ve/scielo.php?script=sci_arttext\&p $\mathrm{id}=\mathrm{S} 1315-95182009000100009$

Mondaca, C. y Gajardo, Y. (2015). Interculturalidad, migrantes y educación. Diálogo andino, (47), 3-6. http://dx.doi.org/10.4067/ S0719-26812015000200001

Mora, D. (2013). Metodología para la investigación de las migraciones. Integra educativa, 6(1), 13-42. http://www.scielo.org.bo/pdf/rieiii/ v6n1/v6n1a02.pdf

Organización Internacional del Trabajo \& Comisión Económica para América Latina y el Caribe. (2017). Coyuntura Laboral en América Latina y el Caribe, (16). http:/www.ilo.org/wcmsp5/groups/ public/---americas/---ro-lima/---sro-santiago/documents/publication/ wcms_553535.pdf

Paredes, R. y Pinto, J. (2009). ¿El Fin de la Educación Pública en Chile? Estudios de economía, 36(1), 47-66.

http://dx.doi.org/10.4067/S0718-52862009000100003

Pavez, I. (2012). Inmigración y racismo: Experiencias de la niñez peruana en Santiago de Chile. Si somos americanos, 12(1), 75-99. http://dx.doi. org/10.4067/S0719-09482012000100004

Penchaszadeh, A. (2015). Ciudadanos extranjeros. Migraciones internacionales, 8(1), 243-248. https://doi.org/10.17428/rmi.v8i1.595 
Picotti, D. (2007). Exigencia histórica de integración e interculturalidad. En: Herceg, J. (comp.). Integración e Interculturalidad (pp. 31-38). Colección idea.

Rodríguez, J. y Arriagada, C. (2004). Segregación Residencial en la Ciudad Latinoamericana. EURE, 30(89), 5-24. http://dx.doi.org/10.4067/S0250-71612004008900001

Romero, A. (2002). Globalización y Pobreza. Ediciones Unariño. https://luiscastellanos.files.wordpress.com/2011/05/romero-albertoglobalizacion-y-pobreza.pdf

Salas, R. (2006). Ética intercultural. Ensayos de una ética discursiva para contextos culturales conflictivos. (Re)lectura del pensamiento latinoamericano. Ediciones UCSH.

Simons, H. (2011). El estudio de caso: Teoría y práctica. Morata.

UNESCO. (2005). Guidelines for inclusion: Ensuring Access to Education for All. http://unesdoc.unesco.org/images/0014/001402/140224e.pdf

\section{Agradecimientos}

Agradecemos como grupo a cada una de las personas que hicieron posible esta investigación: familiares, profesorado, amistades y, en especial, a las/ los/les estudiantes que decidieron compartir sus historias, a todas y todos muchas gracias. 